\title{
Nanorheology of Aqueous Polyethylene Glycol (PEG)
}

\author{
Xueyan Zhang and Steve Granick* \\ Department of Materials Science and Engineering, University of Illinois, Urbana, Illinois 61801 \\ Received September 13, 2001
}

\begin{abstract}
Static and dynamic surface interactions in deionized water were studied between poly(ethylene glycol) (PEG) and an opposed layer of either the same PEG or a highly charged cationic polymer, quaternized polyvinylpyridine (QPVP). The PEG, molecular weight $5000 \mathrm{~g} \mathrm{~mol}^{-1}$, was end-attached to hydrophobized mica by hydrophobic-driven adsorption of the lipid portion (distearoyl-phosphatidylethanolamine) of PEG-lipid diblock copolymers. The QPVP homopolymer, 98\% quaternized and molecular weight $39,000 \mathrm{~g} \mathrm{~mol}^{-1}$, was all owed to adsorb statistically onto mica. Within the scatter of the data the force-distance curves could be fit equally well by the Alexander-de Gennes expression for brush-brush interactions or by exponential decay (decay length $4 \mathrm{~nm}$ ). However, at every film thickness, the shear modulus of the PEG-PEG interface was less than that of PEG-QPVP by an order of magnitude. When the layers were compressed strongly, they remained demonstrably fluid, even in the most strongly compressed state, in the sense that both systems display a clear transition from terminal behavior (viscous forces > elastic forces) to plateau behavior (elastic forces > viscous forces) in the accessible frequency range. This contrasts strongly with the cases of strongly adsorbed nonpolar polymers of which we are aware, but the terminal relaxation time was slower for PEG-QPVP than for PEG-PEG by an order of magnitude. The transition from static to kinetic sliding, i.e., from linear to strongly nonlinear shear response with increasing shear displacement, was examined in the two limits where shear forces were either dominantly viscous (low frequency) or dominantly elastic (high frequency). The yield stress of the PEG-PEG interface was Iarger than that for PEG-QPVP by an order of magnitude; this is attributed to greater interdigitation. It is remarkable that the strong hydrophobic attraction observed between hydrophobized solid surfaces in the absence of polymer was transfigured by a layer of adsorbed polymer to become monotonic repulsion. This comparison of the two systems of adsorbed polymers, symmetric (PEG-PEG) and asymmetric (PEG-QPVP), shows the relative insensitivity of the static-force profiles as compared to dynamic shear responses. The static-force-distance profiles were virtually indistinguishable for the selected experimental conditions but the dynamic shear responses displayed large differences.
\end{abstract}

\section{Introduction}

In the study of dynamic surface interactions in polymeric systems, an initial period of intense interest in interactions in nonpolar solvents is in the process of being expanded to interactions of polymers in aqueous media. Among the water-soluble polymers, poly(ethylene glycol) (PEG) is one of the most interesting because of its potential medical applications. It possesses unusual biocompatibility, nontoxicity, and nonimmunogenicity. ${ }^{1,2}$ PEG has been used extensively for the modification of biomaterial surfaces in attempts to impede protein adsorption and cell adhesion.3,4 Surface modification with PEG has been carried out by various methods, for example, simple physical adsorption, chemical coupling, and graft polymerization. ${ }^{5}$

On the physical side, PEG is a flexible polymer chain. ${ }^{6}$ Many mechanisms to explain its protein resistance have been proposed, among them the large excluded volume of PEG, the configurational entropy, and thickness of grafted layers. ${ }^{3}$ Experimental force-based studies have focused on the static interaction forces between PEO and other biomaterials. ${ }^{3-9}$ The shear behavior has hardly been considered (with the exception of a recent study of friction between adsorbed layers in oil ${ }^{10}$ ) but it is evident that all human body tissues are exposed to aqueous fluid. In addition, PEG is a paradoxical polymer in that, although water-soluble, chains of modest length fail to adsorb to most hydrophilic surfaces except at low $\mathrm{pH}$. However, they do adsorb from water if the surface is hydrophobic.

In this study, the chains were end-attached by the strong adsorption of a hydrophobic tail to better define the configuration of the adsorbed chains. By contrast, when polymers adsorb with equal statistical weight at each segment (the adsorption energy on the order of $k T$ per segment has been quantified for PEO homopolymer in a system similar to the one selected for the present experiments ${ }^{11}$ ), the numerous possible configurations of nearly the same energy result in very slow equilibration and endemic hysteresis of forces. ${ }^{12}$ The strong adsorption in the present case was expected to localize the chains to the specific spot of their end-attachment, despite being accompanied by weaker adsorption of the individual PEO repeat units. The density of surface attachment corresponded to what is known as the "mushroom regime". 13

Using these end-attached PEG chains, we contrast static and dynamic forces in two situations: (a) interactions between PEG layers that were identically prepared; and (b) interactions between PEG on the one side and, on the other side, fully quaternized polyvinylpridine (QPVP). The former case represents polymer interactions in aqueous solution in a good solvent situation. ${ }^{6}$ The latter case, an asymmetric interface, was chosen to represent the interaction of PEG with a highly charged cationic polyelectrolyte with which it is thermodynamically incompatible.

\section{Experimental Section}

Polymers. PEG phospholipid, methoxy PEG linked to distearoyl phosphatidylethanol-amine, was purchased from Shearwater Polymers, I nc. The molecule carries a negative charge due to the ionized phosphate group on the anchoring phospholipid. The molecular weight of the 
PEG portion was 5000 and the distribution of weightaverage to number-average molecular weight was reported to be $M_{w} / M_{n}=1.02$. The polyelectrolyte was poly4-vinylpyridine (Polymer Source, Quebec), quaternized in this laboratory to $98 \%$ (determined by infrared spectroscopy), with ethyl bromide in ethanol solution. The number average molecular weight before quaternization was $M_{n}=34,200 \mathrm{~g} \mathrm{~mol}^{-1}$ (degree of polymerization $n=325$ ) with a polydispersity of $M_{w} / M_{n}=1.23$, and after quaternization the $M_{n}$ was $39,000 \mathrm{~g} \mathrm{~mol}^{-1}$. Static and dynamic forces between symmetric adsorbed layers of QPVP were recently reported by this laboratory. ${ }^{14,15}$

The water used to prepare the solutions was distilled and passed through a Barnstead Nanopure II cleaning system. All experiments were done at $25 \pm 0.5^{\circ} \mathrm{C}$.

Surface Preparation. The PEG-lipid adsorbed to OTE but not to mica. Control experiments verified no adsorption when PEG-lipid was exposed to two opposed sheets of mica.

To prepare mica surfaces for the deposition of PEGlipid, mica was first coated with a self-assembled monolayer of condensed octadecyltriethoxysilane (OTE) using the protocol described previously. ${ }^{16}$ This monolayer is terminated by methyl groups and has been characterized extensively elsewhere. ${ }^{17}$ To check the effectiveness of the monolayer preparation, the OTEcoated surfaces were mounted into the surface-forces apparatus, brought together in dry air and the adhesion was measured. The position of two opposed OTE surfaces in adhesive contact in air was calibrated as zero thickness. Then, the surfaces were separated and a drop of PEG-lipid water solution (0.005-0.02 $\mathrm{mg} \mathrm{mL}^{-1}$ ) was injected, followed by $1-1.5 \mathrm{~h}$ adsorption.

To coat with QPVP, the zero thickness position was calibrated for a single monolayer of OTE (on one side) in contact with bare mica (on the other side) in dry air. The surfaces were separated and a drop of PEG-lipid was injected, followed by $1-1.5 \mathrm{~h}$ adsorption. In the process, PEG-lipid adsorbed to the hydrophobic surface but not to mica. Finally the PEG-lipid solution was replaced by injecting a large amount of $0.1 \mathrm{mg} \mathrm{mL}{ }^{-1}$ QPVP solution. The mica surface is negatively charged in water; therefore, positively charged QPVP segments adsorb strongly. 14,15

Apparatus. The interaction forces and shear properties of the polymer layers at $25^{\circ} \mathrm{C}$ were measured using a surface forces apparatus (SFA) equipped with a device for oscillatory lateral shear. ${ }^{18,19}$ The surfaces were thin sheets of muscovite mica, which were silvered on their backside and glued onto half-cylindrical, fused silica support disks (radius of curvature $\mathrm{R} \approx 2 \mathrm{~cm}$ ) with a thermosetting epoxy glue, EPON 1004F (Shell). The surface separation relative to adhesive contact in dry air was measured by multiple-beam interferometry with an accuracy of $1-2 \AA$. The lower surface was supported on a double cantilever spring with a spring constant of $930 \mathrm{~N} \mathrm{~m}^{-1}$, allowing the force between the surfaces to be determined by monitoring the spring deflection as a function of separation.

The linear shear response of two approaching polymer layers in solution was studied as a function of separation at a frequency of $\mathrm{f}=5.2 \mathrm{~Hz}$ and a displacement amplitude of $\approx 2 \AA$. Strongly compressed layers were also studied in small-amplitude shear experiments in a frequency range of $\mathrm{f}=0.76-180 \mathrm{~Hz}$. To study the transition from rest to kinetic sliding a constant fre quency of $f=2.6$ and $50 \mathrm{~Hz}$ was used with a displacement amplitude up to $150 \AA$.

Shear was induced by applying sinusoi dal voltage to a piezoelectric bimorph element on a device holding the upper surface. The ensuing lateral motion was resisted by the viscoelastic fluid layer confined between the two surfaces, and the complex shear force calculated from the damping and phase shift of the oscillation as detected from the voltage induced in a second, symmetrically placed bimorph element. The response was separated into an elastic force (in phase with the drive) and a dissipative force (out of phase). The elastic and viscous force constants, $g^{\prime} \equiv f_{\text {elastid }} / x$ and $g^{\prime \prime} \equiv f_{\text {viscous }} / x$ (where $\mathrm{x}$ is the displacement amplitude in oscillatory shear deformation), were normalized by the effective contact area multiplied by the film thickness to give effective elastic and viscous shear moduli, G' eff and G" eff. $^{\circ}$ The contact area for the thinnest films (highest compression) was calculated from the measured diameter of the flattened tip of the interference fringes. For thicker films, the effective contact area, $A_{\text {eff }}$ was estimated using the Langbein approximation, $A_{\text {eff }} \approx 2 \pi R D$, where $R$ is mean radius of curvature of the mica surfaces and $D$ is their separation.

Because the air in the chamber of the instrument was exposed to $\mathrm{P}_{2} \mathrm{O}_{5}$ to protect the piezoelectric bimorph elements of the shear device from humidity, rapid evaporation of water occurred from the drop between the surfaces. Each experiment was therefore started immediately after mounting the surfaces, and discontinued after $1.5-2 \mathrm{~h}$ when an evaporation of up to $30 \%$ of the drop could be seen.

\section{Results and Discussion}

Static Forces. Bare Surfaces without Polymer. The strong and long-ranged hydrophobic attraction studied by many researchers using the surface forces apparatus (SFA) and atomic force microscopy (AFM) (for reviews, see refs 22 and 23) was also observed in these experiments in which the mica was rendered hydrophobic by coating with self-assembled OTE monolayers. A detailed analysis of those forces for monolayers of the same kind used in these experiments was presented recently. ${ }^{20}$ In fact, the static-force-distance profiles were found to be almost the same quantitatively when measured with OTE (hydrophobic) on the one side and mica (hydrophilic) on the other. ${ }^{21}$ Figure 1 compares the forcedistance profiles in these two systems. One observes long-ranged attraction, so long-ranged that it began at separations $\approx 0.4 \mu \mathrm{m}$.

The origins of so extraordinarily long-ranged hydrophobic attraction in SFA and AFM experiments has been a mystifying matter for 15 years. Among the many proposed explanations, ${ }^{22,23}$ two of the most prominent concern the possible spontaneous nucleation of vapor bubbles in the vicinity of a hydrophobic surfaces and their subsequent bridging across the intervening gap, and conceivable dipolar attraction between the opposed solid surfaces.

In these experiments with and without adsorbed PEG, the underlying surfaces were the same (hydrophobic monolayers). The variable was the absence or the presence of a thin layer of adsorbed PEG. It is unlikely that hypothesized patterns of in-plane dipoles within a self-assembled monolayer ${ }^{22}$ would be eliminated by the adsorption of PEG. We conclude that the change in sign of forces in the force-distance profile after adsorption 


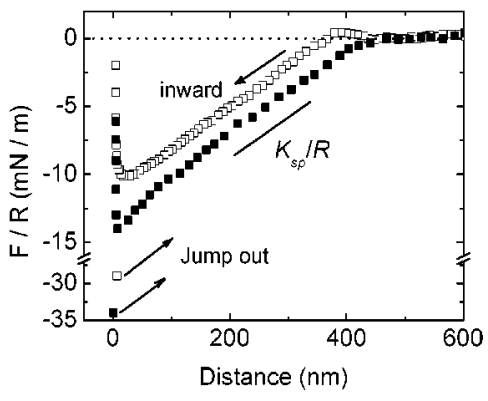

Figure 1. Force-distance profiles in dei onized water between two opposed OTE monolayers (filled symbols) and between OTE on one side and mica on the other side (open symbols). F orces, $F$, normalized by the mean radius of curvature $(R \approx 2$ $\mathrm{cm}$ ) of the crossed cylinders, were measured during approach from static deflection of the force-measuring spring. The pulloff forces ("jump-out") are indicated by respective filled-open symbols and arrows. The approach data follow the straight line with slope $K_{s p} / R$ (drawn separately as a guide to the eye), indicating that they represented a spring instability ("jump in") such that the gradient of attractive force exceeded the spring constant $\left(\mathrm{K}_{\mathrm{sp}}\right), 930 \mathrm{~N} \mathrm{~m}^{-1}$. Data adapted from refs 20 and 21.

of PEG (see next paragraph) does not support the hypothesis that long-range attraction results from the interaction of in-plane surface dipol es. We mention this result, which was tangential to the main concern of this study, because of its significance for understanding origins of "hydrophobic interaction" between extended surfaces.

Surfaces Bearing Adsorbed Polymers. Control experiments showed the QPVP did not adsorb to the hydrophobic surface (OTE) and that PEG-lipid did not adsorb to the adjoining hydrophilic mica. Included in Figure 2 are the force-distance profiles for these same surfaces, the same whose strongly attractive force-distance profiles are shown in Figure 1, but after these polymers were allowed to adsorb (PEG-lipid onto the OTE monolayers and QPVP onto mica). It is evident that in both cases the long-range attraction that is so prominent in Figure 1 was replaced by monotonic repulsion.

The force curves are similar and consist of monotonic repulsion starting at approximately $30 \mathrm{~nm}$. The mean radius of gyration, $R_{G}$, of methyoxy-PEG-DSPE dissolved in water has been estimated to be $6.0 \mathrm{~nm} .{ }^{9}$ The onset of repulsive forces at $\mathrm{D} \approx 4 \mathrm{R}_{\mathrm{G}}$ corresponds to a layer thickness of $\approx 5 \mathrm{R}_{\mathrm{G}}$, which is consistent with findings for statistically adsorbed polymers adsorbed from nonpolar solutions. ${ }^{10,12,24}$ Within the scatter of the limited data, the force-distance curves could be fit equally well by the Alexander-de Gennes expression for brush-brush interactions or by exponential decay (decay length $4 \mathrm{~nm}$ ). This regime of long-rang repulsion ended at a "hard wall" beyond which further compression was not possible. The position of the hard wall was either $3.7 \mathrm{~nm}$ (PEG-QPVP) or $4.7 \mathrm{~nm}$ (PEG-PEG).

As there has been so much past work in the polymer physics community to analyze force-distance profiles of polymer-laden surfaces, our efforts were not concentrated in the direction of varying molecular weight and ionic strength in order to dissect these force-distance relations. The solutions were prepared in deionized water. Although no salt was deliberately added to the solutions, an ionic strength of about $0.6 \mathrm{mM}$ can be estimated from the presence of the counterions of QPVP and PEG-lipid. This would imply a Debye length of about $11 \mathrm{~nm}$, much more than the decay length of $4 \mathrm{~nm}$

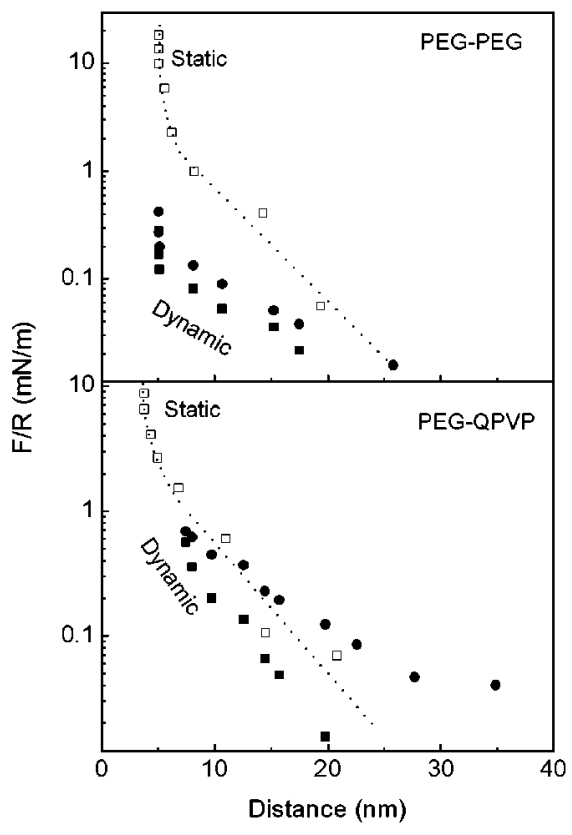

Figure 2. Measurements at variable distance and constant frequency (zero for static forces, $5.2 \mathrm{~Hz}$ and amplitude $2 \AA$ in the shear direction for dynamic shear forces). The forces, normalized by the mean radius of curvature of mica surfaces, $\mathrm{R} \approx 2 \mathrm{~cm}$, are plotted on logarithmic scales as a function of linear surface separation. Top panel, PEG-PEG in deionized water; bottom panel, PEG-QPVP in deionized water. The static forces are denoted by open squares. The elastic and viscous shear forces are solid squares and circles, respectively. The contact of OTE monolayer and bare mica in air is defined as $\mathrm{D}=0$.

shown in Figure 2. The coincidence between the forcedistance relations in the two systems is believed to be a coincidental result of the mol ecular weights that were selected. The common feature of monotonic repulsion is believed to reflect good solvent quality (PEG-PEG) and thermodynamic incompatibility (PEG-QPVP).

Control experiments were performed with a layer of PEG-lipid on one surface and bare mica on the other. If PEG had adsorbed to mica, attraction would have resulted, but the force-distance relation was monotically repulsive instead. Repulsive forces began at $\approx 15$ $\mathrm{nm}$ separation, which is one-half the thickness for two layers of PEG-lipid.

The adsorbed amounts were estimated by measuring the thickness of these layers after removing them from the SFA apparatus, rinsing them, blowing them dry with dry nitrogen, and reinstalling them dry. Assuming the density of $1.0 \mathrm{~g} \mathrm{~cm}^{3}$, the adsorbed amount of PEGlipid was inferred to be about $0.8 \mathrm{mg} \mathrm{m}^{-2}$ and the average spacing between chain ends was $\approx 3.4 \mathrm{~nm}$. For QPVP, the adsorbed amount was about $1.2 \mathrm{mg} \mathrm{m}^{-2}$.

Linear Viscoelastic Shear Measurements. Distance Dependence (Constant F requency of $1.3 \mathrm{~Hz}$ ). Figure 2 al so compares the dynamic shear forces at constant frequency and amplitude to the static forces. One sees that viscous shear forces exceeded the elastic ones except under the strongest compression. Whereas elastic shear forces appeared from the start in the case of PEGPEG, they were immeasurably low for PEG-QPVP until the layers had been compressed by a factor of two. For both systems, viscous shear forces large enough to be detected first appeared at roughly the same separation as static repulsive forces, but slightly lesser separations for the case of PEG-PEG. These measurements hel $p$ to quantify the known reduction of "friction" by PEO.25 


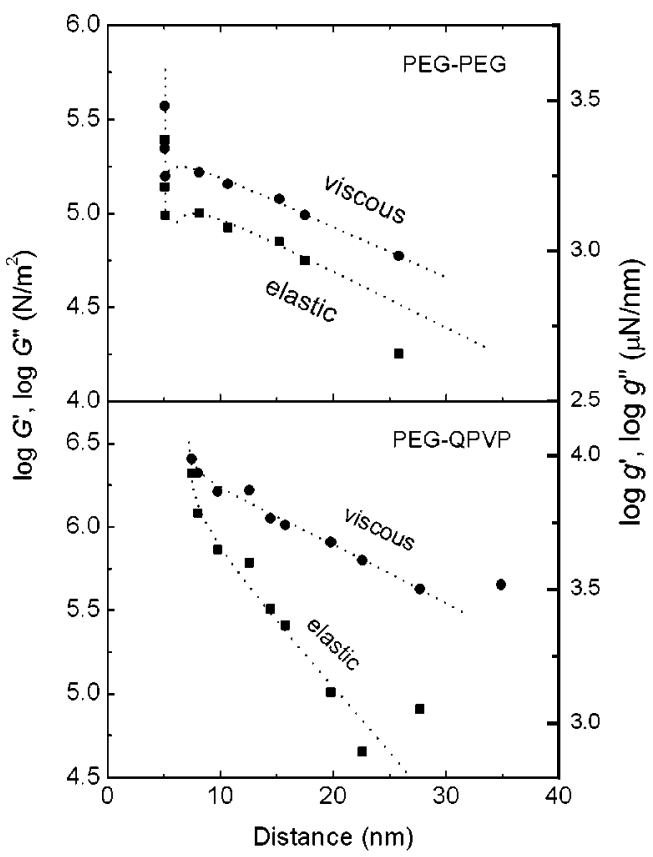

Figure 3. Same dynamic shear data as in Figure 2, except that forces are normalized for strain and effective contact area. On the left-hand ordinate are plotted the logarithmic effective loss modulus, G" (circles) and effective elastic modulus, G' (squares). Top panel, PEG-PEG; bottom panel, PEG-QPVP. On the right-hand ordinate are units showing the equivalent spring constants, out-of-phase with the drive $\left(g^{\prime \prime}\right)$ and in-phase with the drive $\left(g^{\prime}\right)$, with units of force per unit displacement.

After normalizing these raw force measurements by oscillation amplitude for better quantitative comparison (shear forces in the linear response regime are directly proportional to the oscillation magnitude, which was chosen arbitrarily), one obtains the dynamic loss and storage spring constants, g' and g". Further normalization by the estimated contact area gives the effective storage and loss shear moduli, $\mathrm{G}^{\prime}$ and $\mathrm{G}^{\prime \prime}$, respectively. ${ }^{18,19,26}$ In Figure 3, both of these quantities are plotted on semilogarithmic scales against surface separation. The top panel refers to PEG-PEG, the bottom panel to PEG-QPVP. This normalization emphasizes that the magnitudes of the moduli were significantly less for PEG-PEG. In addition, the striking lack of elasticity until the compression was considerable suggests that the case of PEG-QPVP may afford less elastic resistance to shear because of less interpenetration of the opposed polymer layers.

Frequency Dependence (Strong Compression). Figure 4 shows the full frequency dependence of shear responses. The normal force was $F / R \approx 40 \mathrm{mN} \mathrm{m}^{-1}$ but it is better to consider normal pressure, $\mathrm{P}_{\perp} \approx 2 \mathrm{MPa}$, because when the normal force was this large the crossed cylinders flattened to form parallel plates at their apex. The linear dimension of the flattened plates was $\approx 23 \mu \mathrm{m}$. Shear forces have been normalized in two alternative ways, spring constant or effective shear modulus, as in Figure 3. The viscoelastic shear spectra for the two systems, PEG-PEG and PEG-QPVP, evidently differ considerably, in magnitude and position on the frequency scale.

It is well-known from rheology that a system whose dynamical structure is characterized by a dominant relaxation time has constant $\mathrm{G}^{\prime}$ when the drive frequency exceeds this inverse time and that, at lesser frequencies, $\mathrm{G}^{\prime \prime} \sim \omega$ and $\mathrm{G}^{\prime} \sim \omega^{2}$ according to the

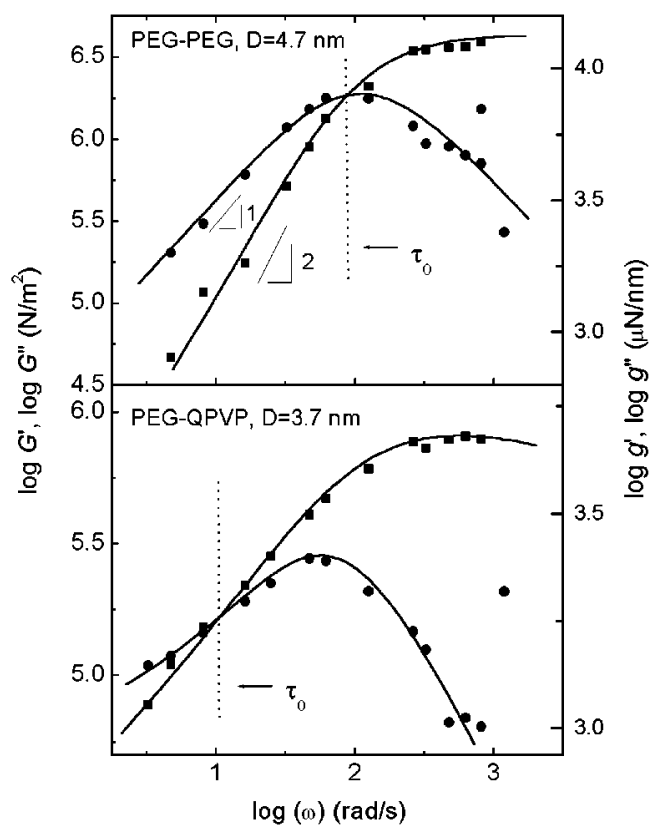

Figure 4. Measurements at constant film thickness and frequency varied. This linear viscoelastic shear spectrum is plotted on log-log scales against logarithmic angular fre quency for strongly compressed PEG-PEG (top panel; thickness $4.7 \mathrm{~nm}$ ) and PE G-QPVP (bottom panel; thickness $3.7 \mathrm{~nm}$ ). On the left-hand axis, solid squares denote storage modulus, $\mathrm{G}^{\prime}(\omega)$; solid circles denote loss modulus, $\mathrm{G}^{\prime \prime}(\omega)$. The right-hand axis shows the equivalent spring constants, $g^{\prime}(\omega)$ and $g^{\prime \prime}(\omega)$, with units of force per unit of shear displacement. Lines with slopes 1 and 2 are drawn as guides to the eye.

Kramers-Kronig relations. ${ }^{26}$ These classic power-law slopes of 1.0 and 2.0 were seen clearly for PEG-PEG they fit more perfectly than do almost all other confined fluids. ${ }^{18,27}$ The somewhat smaller slopes measured for PEG-QPVP (0.9 for $\mathrm{G}^{\prime \prime}(\omega), 1.3$ for $\left.\mathrm{G}^{\prime}(\omega)\right)$ indicate a distribution of relaxation times over the accessible frequency scale.

The frequency at which the crossover of $\mathrm{G}^{\prime}$ and $\mathrm{G}^{\prime \prime}$ occurs can be used to define the system's terminal relaxation time, $\tau_{0}=2 \pi / \omega_{0}(0.07 \mathrm{~s}$ for PEG-PEG, $0.6 \mathrm{~s}$ for PEG-QPVP), although $\tau_{\mathrm{o}}$ is more cleanly defined for the former system than the latter, whose frequency dependence indicates a distribution of processes even in the regime where $\mathrm{G}^{\prime \prime}(\omega)>\mathrm{G}^{\prime}(\omega)$. The dynamic shear responses of both systems show a highly sluggish interfacial relaxation behavior that nonetheless was predominantly viscous (liquidlike) when the frequency was sufficiently low.

These liquidlike responses contrast strongly with the behavior of confined nonpolar polymers. These are "solid", with G' $(\omega) \gg G^{\prime \prime}(\omega)$ over the accessible frequency scale, when they are compressed to a si milar extent.18,27

Transition from Static to Kinetic Sliding. When the shear amplitude increases beyond a certain value, the interfacial fil $\mathrm{m}$ is nolonger able to hold the induced shear stresses, but rather transits from static to kinetic motion. We now describe, for strongly compressed films, this regime of strongly nonlinear shear responses.

Previously this laboratory proposed a rate-criterion for stick-slip motion: 28 flow is expected to be fluidlike if the forcing frequency was less than the inverse natural relaxation time (shear-thinning), whereas stick-slip is expected if the forcing frequency was so high that the system was deformed faster than it could keep up. In systems where the terminal relaxation falls outside the 


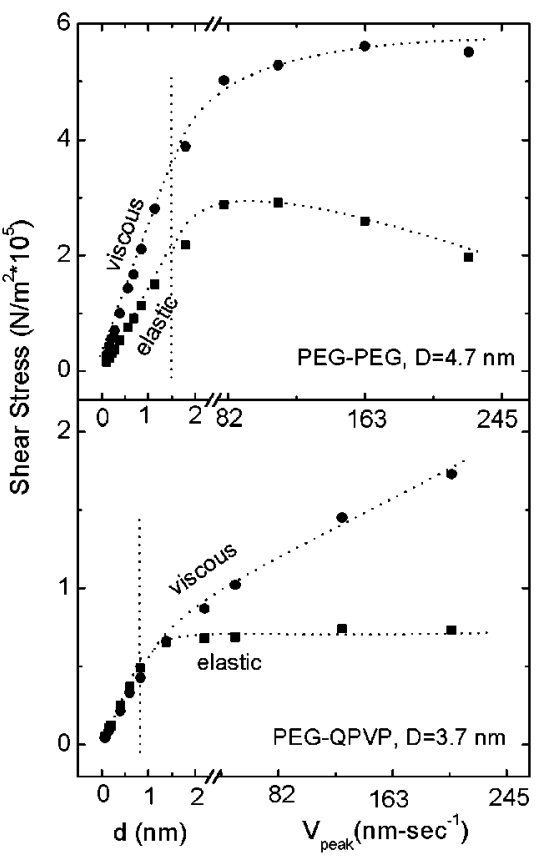

Figure 5. In this figure the shear frequency is less than the inverse terminal relaxation time identified in Figure 4 . The amount of shear, in the regime of linear response, as presented as a function of increasing displacement amplitude (d). For larger displacements it is presented as a function of increasing peak velocity, $\mathrm{v}_{\text {peak }} \approx \omega \mathrm{d}$, because the surface were sliding. The forces have been normalized by contact area to give elastic shear stress (squares) and viscous shear stress (circles), both evaluated at the fundamental frequency. The top panel refers to PEG-PEG (thickness $4.7 \mathrm{~nm}$, shear frequency $2.6 \mathrm{~Hz}$ ) and the bottom panel to PEG-QPVP (thickness $3.7 \mathrm{~nm}$, shear frequency $5.2 \mathrm{~Hz}$ ). If the reader wishes to estimate the effective friction coefficient, note that the static normal force amounted to the normal pressure of $2 \mathrm{MPa}$. The dotted vertical line near the origin is a guide to the eye showing the point at which linear response was lost.

accessible frequency range, this notion cannot be tested. But the present systems were good candidates.

F or the case where the linear shear responses were predominantly viscous because the forcing frequency was low, Figure 5 shows the shear stress as a function of shear displacement. Here the nonlinear forces were evaluated at the same frequency as the forcing drive; el sewhere we have evaluated critically the validity of this lack of attention to harmonic frequencies present in nonlinear responses. ${ }^{27}$ The top panel denotes PEGPEG $(2.6 \mathrm{~Hz})$, the bottom panel PEG-QPVP $(5.2 \mathrm{~Hz})$.

A striking difference between the systems is that the PEG-PEG system was able to withstand a consi derably larger shear stress, both viscous and elastic, than PEGQPVP. This is consistent with the hypothesis, al ready suggested from the thickness dependence of shear forces, that PEG-QPVP interfaces were less interdigitated. A striking commonality between the systems is that both became nonlinear when the shear displacement was a substantial fraction of the total film thickness: $1.5 \mathrm{~nm}$ (PEG-PEG) and $0.8 \mathrm{~nm}$ (PEG-QPVP). A second, common point is that the motion was continuous; the shear stresses changed monotonically with shear displacement, without any discontinuity.

For the case where the linear responses were predominantly elastic because the forcing frequency was large, Figure 6 shows the shear stress plotted against shear displacement. Again, the nonlinear forces were evaluated at the same frequency as the forcing drive.

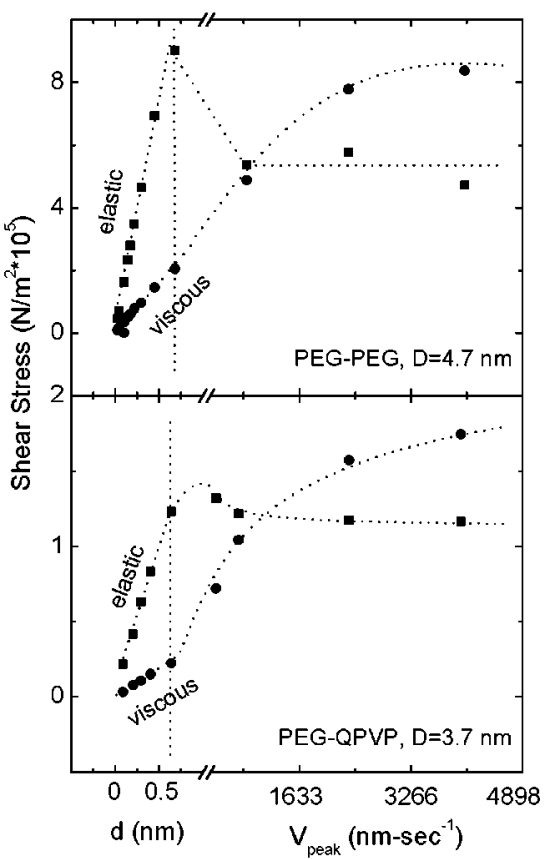

Figure 6. In this figure, the shear frequency exceeds the inverse terminal relaxation time identified in Figure 4 . The amount of shear, in the regime of linear response, as presented as a function of increasing displacement amplitude (d). For larger displacements it is presented as a function of increasing peak velocity, $v_{\text {peak }} \approx \omega \mathrm{d}$, because the surface were sliding. Symbols are same as in Figure 5. N ote the el astic yiel d stress, which is the maximum elastic shear stress. If the reader wishes to estimate the effective friction coefficient, note that the static normal force amounted to the normal pressure of 2 MPa. The dotted vertical line near the origin is a guide to the eye showing the point at which linear response was lost.

The top panel denotes PEG-PEG, the bottom panel PEG-QPVP ( $52 \mathrm{~Hz}$ for both systems). We observed that the linear-response elastic stresses grew rapidly up to a point where the system failed catastrophically: the elastic stress passes through a maximum and when the deformation was still larger, the viscous forces dominated the elastic ones. This is analogous to yielding of a glass or a gelled colloidal suspension.

Upon comparing the systems in their response to this higher forcing frequency, it is obvious that again, the PEG-PEG system sustained considerably higher stresses than the PEG-QPVP system, again consistent with the hypothesis of greater interdigitation. The shear stress at yield was much higher than at lower frequency. The deformation at yield was a significant fraction of the film thickness $(\approx 0.6 \mathrm{~nm}$ for both PEG-PEG and PEGQPVP). After yield, the elastic shear stress for PEGPEG dropped by $45 \%$.

\section{Conclusions}

It is striking to observe that the presence of a single adsorbed layer of flexible polymer chain transformed the sign of the long-range forces between the solid surfaces studied here: from strongly attractive in absence of the polymer layers, to monotonically repulsive when they were present.

This comparison of the two systems of adsorbed polymers, symmetric (PEG-PEG) and asymmetric (PEGQPVP), shows the relative insensitivity of the staticforce profiles as compared to dynamic shear responses. The static force-distance profiles were virtually indistinguishable for the selected experimental conditions, 
but the dynamic shear responses displayed large differences. The direction of the differences was to suggest larger interdigitation of the opposed layers in the case of PEG-PEG. This is reasonable physically, because PEG is thermodynamically incompatible with QPVP. According to the degree of thermodynamic incompatibility, the systems displayed very different (larger or smaller) shear stresses at the point of elastic yield.

Another striking difference is the demonstrably fluid character of these adsorbed layers, even in the most strongly compressed state. This conclusion comes from the linear-response dynamic mechanical responses, which for both systems display a clear transition from terminal behavior (viscous forces > elastic forces) to plateau behavior (elastic forces $>$ viscous forces) in the accessible frequency range. This contrasts strongly with the cases of strongly adsorbed nonpolar polymers of which we are aware.

Acknowledgment. This work was supported by the taxpayers of the United States through the National Science Foundation with the aid of facilities provided by the U.S. Department of Energy, Division of Materials Science through grant DE FG02-91ER45439 to the Fre derick Seitz Materials Research Laboratory at the University of Illinois at Urbana-Champaign.

\section{References and Notes}

(1) Poly(ethyleneglycol): Chemistry and Biological Application; Harris, J. M., Zalipsky, S., Eds.; American Chemical Society: Washington, DC, 1997.

(2) Elbert, L.; Hubbell, J. A. Annu. Rev. Mater. Sci. 1996, 26, 365.

(3) Zhu, B.; Eurell, T.; Gunawan, R.; Leckband, D. J . Biomed. Mater. Res. 2001, 56, 406; and references therein.

(4) Ostune, E.; Chapman, R. G.; Holmlin, R. E.; Takayama, S.; Whitesides, G. M. Langmuir 2001, 17, 5605; and references therein.
(5) Gong, X.; Dai, L.; Griesser, H. J .; Mau, A. W. H. J . Polym. Sci. B. 2000, 38, 2323; and references therein.

(6) Faraone, A.; Magazù, S.; Maisano, G.; Migliardo, P.; Tettamanti, E.; Villari, V. J . Chem. Phys. 1999, 110, 1801.

(7) KuhIL, T. L.; Leckband, D. E.; Lasic, D. D.; Israelachvili, J . N. Biophys. J . 1994, 66, 1479.

(8) Sheth, S. R.; Efremova, N.; Leckband, D. E. J . Phys. Chem. B. 2000, 104, 7652.

(9) Efremova, N. V.; Sheth, S. R.; Leckband, D. E. Langmuir 2001, 17, 7628

(10) Raviv, U.; Tadmor, F.; Klein, J . J . Phys. Chem. B 2001, 105, 8125.

(11) Sukhishvili, S. A.; Chen, Y.; Müller, J .; Gratton, E.; Schweizer, K. S.; Granick, S. Nature 2000, 406, 146.

(12) Klein, J .; Luckham, P. F. Macromolecules 1984, 17, 1041.

(13) Watanabe, H.; Kilbey, S. M.; Tirrell, M. Macromolecules 2000, 33, 9146; and references therein.

(14) Ruths, M.; Sukhishvili, S. A.; Granick, S. J . Phys. Chem. B. 2001, 105, 6202.

(15) Sukhishvili, S. A.; Dhinojwala, A.; Granick, S. Langmuir 1999, 15, 8474.

(16) Kessel, C. R.; Granick, S. Langmuir 1991, 7, 532.

(17) Peanasky, J .; Schneider, H. M.; Kessel, C. R.; Granick, S. Langmuir 1995, 11, 953.

(18) Demirel, A. L.; Granick, S. J . Chem. Phys. 2001, 115, 1498.

(19) Peachey, J .; Van Alsen, J .; Granick, S. Rev. Sci. Inst. 1991, $62,463$.

(20) Zhang, X.; Zhu, Y.; Granick, S. J . Am. Chem. Soc. 2001, 123, 6736.

(21) Zhang, X.; Zhu, Y.; Granick, S. Science 2002, 295, 663.

(22) Spalla, O. Current Opinions in Colloid Interface Sci. 2000, 5, 5; and references therein.

(23) Tyrrell, J. W.G.; Attard, P. Langmuir 2002, 18, 160.

(24) Hu, H-W.; Granick, S. Macromolecules 1990, 23, 613.

(25) Gadd, G. E. Nature 1968, 217, 1040.

(26) Ferry, J . D. Viscoelastic Properties of Polymers, 3rd Ed.; Wiley: New York, 1980.

(27) Granick, S.; Hu, H.-W. Langmuir 1994, 10, 3857.

(28) Dhinojwala, A.; Cai, L.; Granick, S. Langmuir 1996, 12, 4537. MA011626S 